\title{
Archéopages
}

Archéopages Archéologie et société

40 | 04-07/2014

Villages

\section{Premier hameau de l'âge du Bronze final en Bretagne}

\section{Mélanie Levan}

\section{(2) OpenEdition}

1 Journals

Édition électronique

URL : https://journals.openedition.org/archeopages/595

DOI : 10.4000/archeopages.595

ISSN : 2269-9872

\section{Éditeur}

INRAP - Institut national de recherches archéologiques préventives

\section{Édition imprimée}

Date de publication : 1 juin 2015

Pagination : 48-49

ISSN : $1622-8545$

\section{Référence électronique}

Mélanie Levan, « Premier hameau de l'âge du Bronze final en Bretagne », Archéopages [En ligne], 40 04-07/2014, mis en ligne le 01 juillet 2016, consulté le 23 janvier 2022. URL : http:// journals.openedition.org/archeopages/595; DOI : https://doi.org/10.4000/archeopages.595 

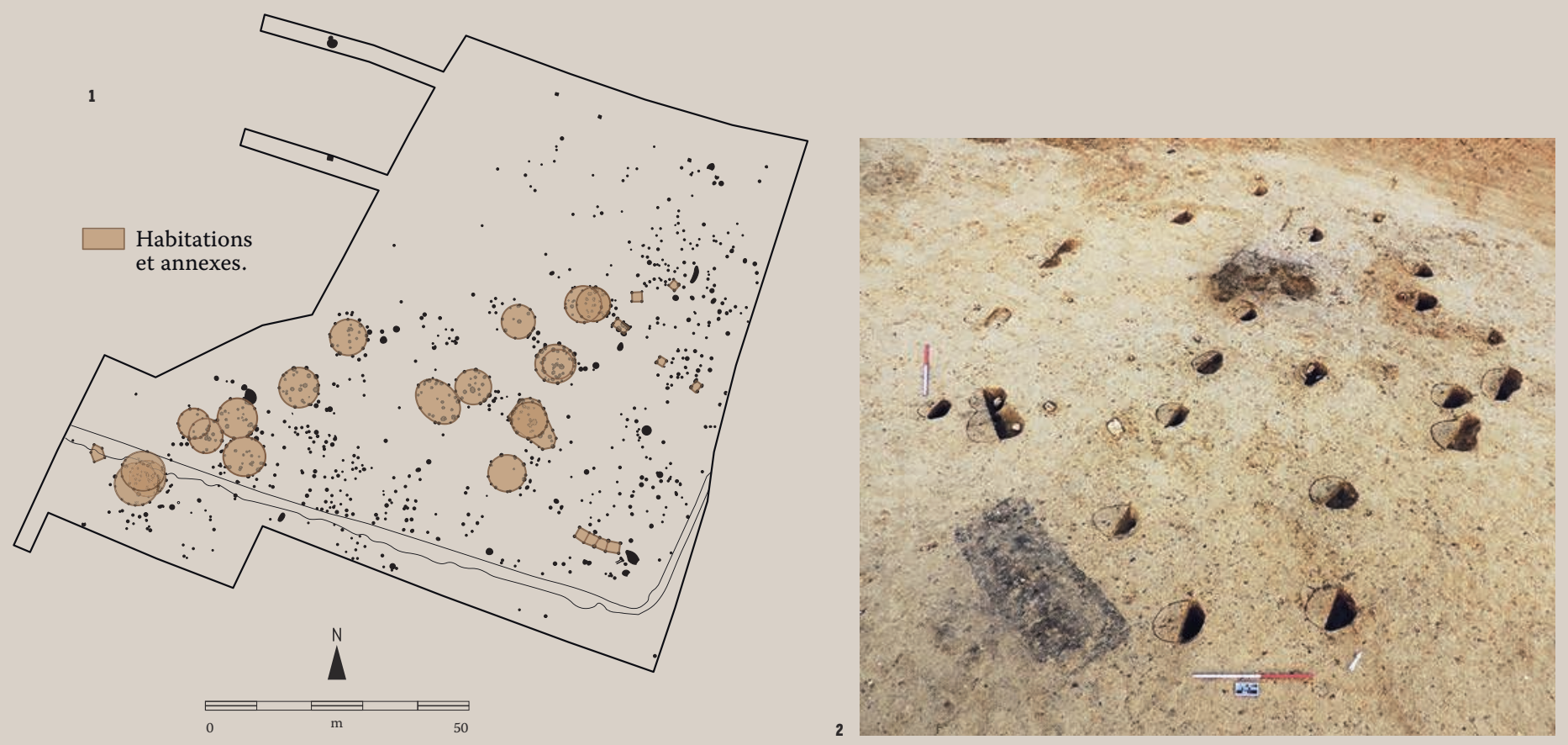

\section{Premier}

\section{hameau de l'âge du Bronze final} en Bretagne
L'étude en cours porte sur une importante zone d'habitat fréquentée à l'âge du Bronze final à Caudan (Morbihan). Cette fouille succède à un diagnostic de $30 \mathrm{ha}$ mené par Fddy Roy en 2012 (Roy, 2012), en amont d'un aménagement de ZAC. À une centaine de mètres de l'occupation protohistorique, une installation enclose (ferme ?) du haut Moyen Âge a été fouillée par une deuxième équipe de l'Inrap sous la direction de Françoise Le Boulanger (étude à venir). Situé à une dizaine de kilomètres de Lorient et du littoral atlantique, le site s'inscrit dans le prolongement surélevé des plaines côtières, dans un paysage modelé de petites collines et encadré par les rivières du Scorff et du Blavet. Dans un contexte hydrographique régional assez dense, il s'implante entre la vallée encaissée du ruisseau du Plessis, à l'ouest, et le ruisseau de Kergoal son affluent, à l'est. L'occupation se développe sur le rebord d'un petit sommet culminant à $61 \mathrm{~m}$ NGF. Installée sur l'amorce du versant sud, en surplomb d'un talweg, sa position offre un panorama dégagé.

Au regard du plan général et des indices livrés au diagnostic, il semble que le gisement s'étende au-delà de l'hectare décapé. Les premières datations ${ }^{14} \mathbf{C}$ révèlent une occupation s'échelonnant sur près de 600 ans, entre les xrv et virr avant notre ère, soit durant tout le Bronze final. Un nettoyage manuel extensif a permis de mettre en évidence plus de 1100 trous de poteau. Vingt bâtiments d'habitation, la plupart de plan circulaire, ont été identifiés. Se développant sur un axe nord-est/sud-ouest, ces maisons présentent des dimensions standard, entre 6 et $8 \mathbf{m}$ de diamètre [ill. 1 et 2]. Les porches conservés montrent la systématisation de leur orientation vers le sud-est. Les premières observations suggèrent des techniques de construction assez variées, même entre des bâtiments potentiellement synchrones : structuration du porche d'entrée, présence ou non d'un élément central. À ces constructions s'adjoignent des petites dépendances, interprétées traditionnellement comme des annexes agricoles, construites sur quatre poteaux ou plus. Des graines ont pu être prélevées dans certains de ces creusements. L'étude carpologique en cours ${ }^{1}$ permettra peut-être de restituer avec plus de précision les pratiques agricoles pour cette période, encore mal renseignées dans la région. Au regard du plan général, il est possible d'envisager plusieurs esquisses de palissades et délimitations légères, matérialisant d'éventuels petits espaces de pacage. La plupart des vestiges liés à l'activité agropastorale ne semblent pas se développer entre les bâtiments d'habitation. Leurs implantations en marge de celles-ci auraient été privilégiées. Associé à cette activité pastorale, le domaine de l'artisanat est représenté par des éléments de tissage: fusaïoles et pesons. Les fosses dépotoirs riches en mobilier, retrouvées fréquemment sur les sites d'habitat de cette période, sont absentes de la zone décapée.

Les datations ${ }^{14} \mathbf{C}$ réalisées restituent une occupation continue. Plus qu'un 


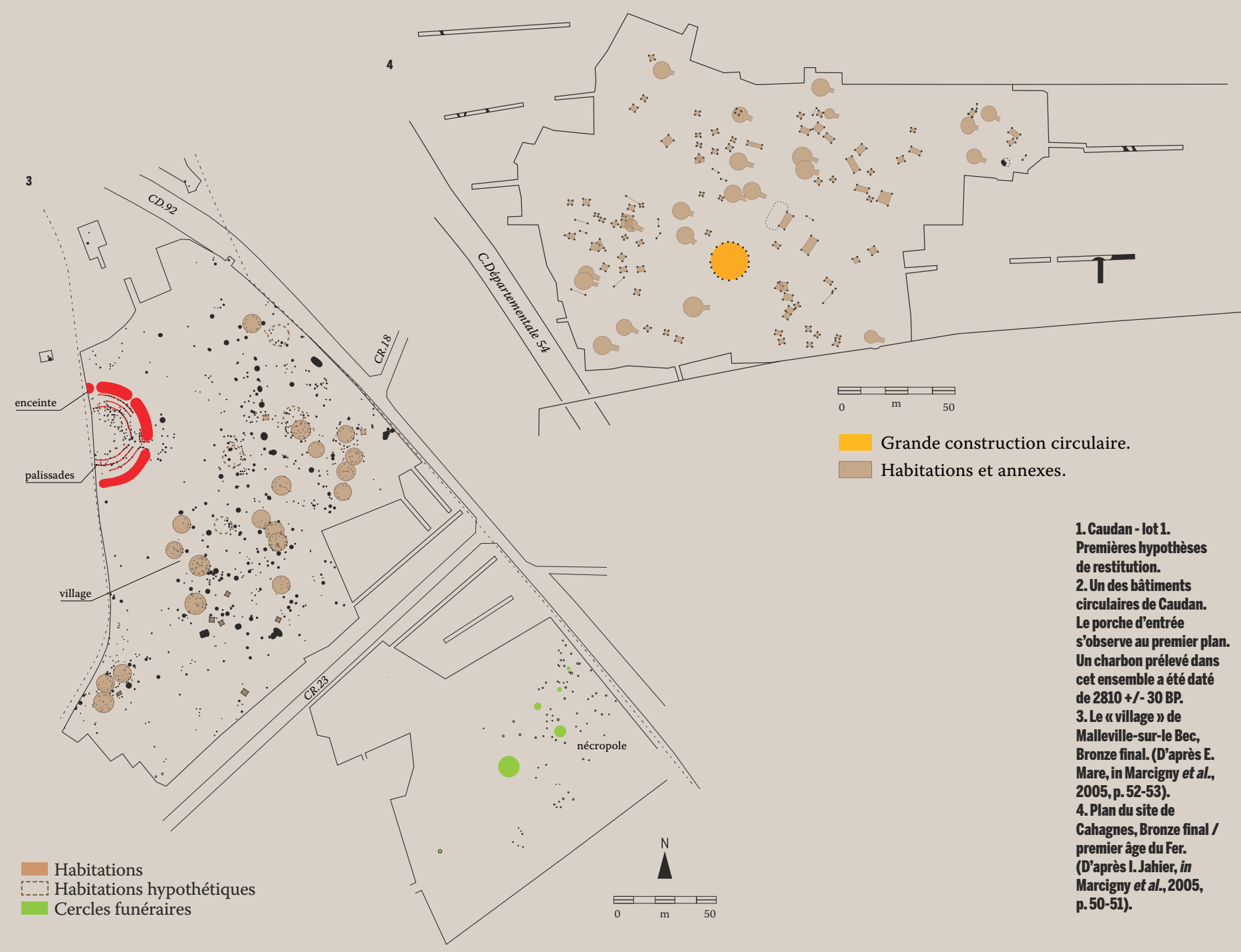

phénomène de concentration important, il s'agirait très probablement d'un regroupement de quelques unités sur une trame resserrée, sans forme hiérarchique apparente. Si la densité d'occupation s'accélère à partir de 1000 avant notre ère, les habitations coexistantes ne semblent pas excéder un petit nombre. La faible profondeur des trous de poteaux, ne dépassant parfois guère plus de quelques centimètres, suggère l'érection de bâtiments assez légers, pouvant correspondre à l'installation d'une génération. Si des éléments céramiques attribuables au Bronze ancien et au Campaniforme attestent une attractivité du secteur depuis des périodes plus anciennes, on peut se poser la question des raisons du choix de cet espace, particulièrement privilégié à la fin de l'âge du Bronze. La topographie singulière ou la proximité d'un élément symbolique, ou du moins catalyseur, pourraient en expliquer la motivation. La répartition particulière des habitations autour du sommet de la colline, emplacement généralement favorisé pour l'implantation des vestiges funéraires, pourrait corroborer cette dernière hypothèse.
Le site de Caudan, par sa densité de vestiges, l'organisation de l'espace et les techniques de constructions architecturales, rappelle les exemples répartis de part et d'autre de la Manche, définissant la culture Manche-mer du Nord. Les sites normands de Cahagnes (Calvados) (I. Jahier, 1996, 2005), et Maleville-sur-le-Bec (Eure) (F. Mare, 2010) en sont les meilleures comparaisons [ill. 3 et 4]. À Caudan, l'absence de fossés délimitateurs définit une occupation ouverte, où la concentration des unités, sans forme hiérarchique apparente, formule l'hypothèse d'un hameau. Ce mode d'organisation se distingue à ce titre des fermes isolées qui composaient jusqu'à présent l'essentiel du corpus breton, parmi lequel on peut citer, pour la période du Bronze final, le gisement de Plédéliac (Finistère), " Nord du bourg " (F. Nicolas, 2011) et celui de La Mézière (IIle-et-Vilaine), « Les Lignes de la Gonzée " (S. Blanchet, à paraître). Ces nouvelles données induisent d'autres questionnements sur les modes d'occupation dans la péninsule armoricaine.

\author{
Premières hypothèses \\ de restitution. \\ . Un des batiments \\ Le porche d'entrée \\ s'observe au premier plan \\ Un charbon prélevé dans \\ de $2810+/-30$ BP. \\ 3. Le " village $)$ de \\ (D'après E. \\ 2005, p. 52-53). \\ 4. Plan du site de \\ Cahagnes, Bronze final / \\ premier âge du Fer. \\ Marcigny et al.,2005, \\ p. 50-51).
}

Références bibliographiques

BLANCHET S. (DIR.), à paraître : La Mézière (35), ZAC des Lignes de la Gonzée, Rapport final d'opération de fouille, Inrap Grand-Ouest.

JAHIER I. (DIR.), 1996 : Cahagnes ZS 93 «Benneville », A84, Site:14.120.005.AH, Rapport de fouille de sauvetage urgent, SRA de Basse-Normandie, Caen, 84 p.

JAHIER I., 2005 : « Le village de Cahagnes (Calvados) », in MARCIGNY C. (DIR.), La Normandie à l'aube de l'Histoire : les découvertes archéologiques de l'âge du Bronze, 2300-80o av. J.-C., Paris, Somogy éditions d'art.

MARE E. (DIR.), 2010 : Le village de l'âge du Bronze final du Buisson-du-Roui à Malleville-sur-le-Bec (Eure), Rapport final d'opération, Série « Construction de l'Autoroute A28 nord (Alençon-Rouen) », Inrap, Drac de Haute-Normandie, SRA, Rouen, $180 \mathrm{p}$.

Nicolas E., 2011: Plédéliac, Côtes d'Armor; Nord du bourg. Deux bâtiments de l'âge du Bronze, Rapport final d'opération de fouille, Inrap Grand-Ouest, 75 p.

Roy E., 2012, Caudan, Morbihan, lieu dit «LennSec'h». Découverte d'une vaste zonz d'occupation datée de l'âge du Bronze, à Caudan dans le Morbihan, Rapport final d'opération, Inrap/SRA Bretagne, 128p. 